\title{
AID/APOBEC deaminases and cancer
}

\author{
Stefan RebhandI ${ }^{1,2}$, Michael Huemer ${ }^{1,2}$, Richard Greil $^{1,2}$, Roland Geisberger $^{1,2}$ \\ ${ }^{1}$ Department of internal Medicine III with Hematology, Medical Oncology, Hemostaseology, Infectious Diseases, Rheumatology, \\ Oncologic Center, Laboratory for Immunological and Molecular Cancer Research, Paracelsus Medical University Salzburg, \\ Austria \\ ${ }^{2}$ Salzburg Cancer Research Institute, Salzburg, Austria \\ Correspondence to: Roland Geisberger, email: r.geisberger@salk.at
}

Keywords: deamination, cancer, AID, APOBEC3, mutation signature, mutation cluster, kataegis

Received: February 19,2015 Accepted: April 01,2015 Published: April 28, 2015

This is an open-access article distributed under the terms of the Creative Commons Attribution License, which permits unrestricted use, distribution, and reproduction in any medium, provided the original author and source are credited.

ABSTRACT:

Mutations are the basis for evolution and the development of genetic diseases. Especially in cancer, somatic mutations in oncogenes and tumor suppressor genes alongside the occurrence of passenger mutations have been observed by recent deepsequencing approaches. While mutations have long been considered random events induced by DNA-replication errors or by DNA damaging agents, genome sequencing led to the discovery of non-random mutation signatures in many human cancer. Common non-random mutations comprise DNA strand-biased mutation showers and mutations restricted to certain DNA motifs, which recently have become attributed to the activity of the AID/APOBEC family of DNA deaminases. Hence, APOBEC enzymes, which have evolved as key players in natural and adaptive immunity, have been proposed to contribute to cancer development and clonal evolution of cancer by inducing collateral genomic damage due to their DNA deaminating activity. This review focuses on how mutagenic events through AID/APOBEC deaminases may contribute to cancer development.

\section{INTRODUCTION}

Genomic DNA, composed of the four deoxynucleotides $\mathrm{dA}, \mathrm{dC}, \mathrm{dG}$ and $\mathrm{dT}$, is being damaged constantly and therefore requires continuous DNA repair $[1,2]$. In particular, cytosine and 5-methylcytosine are susceptible to spontaneous hydrolytic deamination generating $\mathrm{dU}: \mathrm{dG}$ and $\mathrm{dT}: \mathrm{dG}$ mismatches leading to somatic and germline mutations, if not or improperly repaired [2,3]. Aside from spontaneous cytosine hydrolysis, uracils can also be introduced into DNA by polymerase-catalyzed mis-incorporation of dUTP instead of dTTP or through cytidine deaminase-mediated deamination of $\mathrm{dC}$ residues present in the DNA [4]. In higher eukaryotes, the $\mathrm{dC}$ deaminases all belong to the AID/APOBEC family of deaminases. In this review, the role of aberrant AID/APOBEC activity for cancer development is described.

The AID/APOBEC family of deaminases

The AID/APOBEC family of proteins represents a group of cytidine deaminases that fulfills diverse physiological functions [5,6]. The protein family comprises eleven members in humans: AID and APOBEC1 (genes located on chromosome 12), APOBEC2 (gene located on chromosome 6), seven APOBEC3 proteins (APOBEC3A, APOBEC3B, APOBEC 3C, APOBEC3D, APOBEC $3 F$, APOBEC $3 \mathrm{G}$, APOBEC $3 \mathrm{H}$; genes located on chromosome 22) and APOBEC4 (gene located on chromosome 1) [7-12]. AID and APOBEC2 are considered the ancestral members of the AID/APOBEC family [5], whereas APOBEC1 and APOBEC3 appeared later as AID derivatives [5]. APOBEC3 proteins are detectable exclusively in mammals $[5,13]$. The gene copy number is species-specific, mice have a single APOBEC3 gene, while pigs (2), sheep (3), cattle (3), cats (4), horses (6) and primates (at least 7 APOBEC 3 genes) have multiple copy numbers $[5,10,13,14]$. All members of the AID/APOBEC family share at least one zinc-binding catalytic domain with the consensus amino acid sequence $\mathrm{H}-\mathrm{X}-\mathrm{E}-\mathrm{X}_{23-28}-\mathrm{P}-\mathrm{C}-\mathrm{X}_{2-4}-\mathrm{C}$ (X stands for any amino acid) $[10,14,15]$.

The first member to be identified and characterized was APOBEC1, which deaminates mRNA for apolipoprotein B at cytosine ${ }^{6666}$ to uracil [16-18]. The resulting premature stop codon generates apoB- 48 , which 
mediates uptake of dietary lipid from the intestine [1618]. Additional mRNA targets for APOBEC1 have been described recently, where the interaction occurs at AUrich sequence elements in 3' UTRs and regulates mRNA stability $[19,20]$. Beside its role as an RNA editing enzyme [16], APOBEC1 is also capable of editing DNA in bacterial assays [21].

APOBEC2 was the second AID/APOBEC family member to be identified [9]. It is expressed in skeletal muscle and heart [9], but does not exhibit mutagenic activity in yeast and bacterial assays [22]. Although its precise physiological role has not been identified yet, APOBEC2 expression appears to be crucial for muscle development [23].

The group of APOBEC3 enzymes was first characterized by Jarmuz and co-workers as paralogs of APOBEC1 [10] and comprises seven proteins in humans (APOBEC3A-D, APOBEC3F, APOBEC3G, APOBEC3H) [12]. According to the number of the zinc-finger domains, APOBEC3 proteins can be classified in two groups: APOBEC3A, APOBEC3C and APOBEC3H have a single zinc-finger domain, whereas APOBEC3B, APOBEC3D, APOBEC3G and APOBEC3F harbor two zinc-finger domains $[14,15]$. APOBEC3 enzymes play an important role in innate immunity by inhibiting retroviruses through deamination of retroviral DNA intermediates [6]. In 2002, APOBEC3G was identified as the responsible factor for the restriction of Vif-deficient HIV-1 [24]. The retroviral restriction by APOBEC3 is counteracted by the Vif gene in human and rodent immunodeficiency viruses, as the Vif protein leads to degradation of APOBEC3 protein [24]. APOBEC3G is packaged into the HIV virions in an infected cell and in further consequence deaminates cytosines in the nascent DNA strand during viral reverse transcription in the newly infected target cell $[6,15]$. The uracil containing first strand then functions as template for second strand synthesis and thereby results in $\mathrm{dG}>\mathrm{dA}$ hypermutations, which affect the viability of the virus and its ability to integrate into the host genome $[6,15]$. The Vif protein triggers polyubiquitination and subsequent degradation of APOBEC $3 \mathrm{G}$ before virion incorporation [6]. But also APOBEC3B and APOBEC3F have been shown to have antiretroviral activity, at least for HIV-1 [25-28]. Aside from HIV, APOBEC3 proteins are also involved in defense against other viruses like human T-cell lymphotropic virus, hepatitis B virus, hepatitis $\mathrm{C}$ virus (HCV), human papillomavirus (HPV) and human herpesviruses [15]. Furthermore, APOBEC3 enzymes can restrict the movement of non-LTR and LTR retrotransposable elements, including long interspersed nuclear elements (LINEs) and short interspersed nuclear elements (SINEs) [29]. APOBEC3 proteins display distinct subcellular localization: APOBEC3B localizes to the nucleus [30-32], APOBEC3D, APOBEC3F and APOBEC3G are present in the cytoplasm [30,32], while APOBEC3A, APOBEC3C and APOBEC3H localize to both, nucleus and cytoplasm [32,33]. APOBEC3 proteins also mediate the clearance of exogenous doublestranded DNA (dsDNA) in human cells, as in a recent study APOBEC3A deaminated up to $97 \%$ of cytosines in foreign DNA leading to degradation, whereas genomic DNA remained unaffected [34]. The preferred sequence context for APOBEC3-mediated deamination is the TCW motif $(\mathrm{W}=\mathrm{A}$ or $\mathrm{T})$, in contrast to the APOBEC3G hot spot motif $\mathrm{CC}$ and the AID hot spot motif WRCY $(\mathrm{R}=$ $\mathrm{A}$ or $\mathrm{G}, \mathrm{Y}=\mathrm{C}$ or $\mathrm{T}$ ) [35-41]. AID/APOBEC deaminases need single-stranded DNA (ssDNA) as substrate [6,4147]. Quantitative profiling of APOBEC 3 mRNA in 20 different human tissues, human T-cell lines and bulk leukocytes as well as leukocyte subsets demonstrated that APOBEC3 genes are expressed broadly and constitutively and that expression is not limited to immune cells [48]. However, APOBEC2 $2^{-/}$and APOBEC3 $3^{-/}$knockout mice $^{-}$ display no obvious phenotype, suggesting that these genes are inessential for mouse development, viability or fertility [49]. Interestingly, recent work from Halemano and coworkers showed evidence that mouse APOBEC3 mutates immunoglobulin heavy chain variable genes $(\mathrm{IgV})$ during retroviral infections, demonstrating thereby a previously unidentified function of APOBEC3 in generating virusspecific neutralizing antibodies and highlighting a new mechanism for antibody diversification in vivo [50]. In addition, APOBEC3 has also been attributed a role in double-strand break (DSB) DNA repair by nonhomologous end joining (NHEJ) [51,52]. Expression of APOBEC3G in lymphoma cells associates with efficient DSB repair, whereas inhibition of APOBEC $3 \mathrm{G}$ expression or deamination activity results in impaired joining of DSBs by NHEJ, implying a prosurvival role of APOBEC3 in lymphoma cells [51].

APOBEC4, the newest AID/APOBEC family member, is primarily expressed in testicles [11]. Its function is still unknown. Bacterial and yeast assays did not reveal mutagenic activity [22].

The ssDNA deaminating enzyme AID, which is primarily expressed in germinal center (GC) B cells, is the key deaminating enzyme for antigen-dependent antibody diversification through somatic hypermutation (SHM) and class switch recombination (CSR) [53-55]. There is a distinct evolutionary link between the vertebrate immune system and AID. This molecule is found in bony fish and cartilaginous fish, which are the first life forms to have an adaptive immune system [56]. AID deaminates $\mathrm{dC}$ thereby generating $\mathrm{dU}$ within the immunoglobulin (Ig) locus $[53,55,57,58]$. Since uracil base pairs with adenine, unrepaired uracils on the one hand lead to $\mathrm{dC}>\mathrm{dT}$ transition mutations after DNA replication, whereas error prone DNA repair of uracil lesions on the other hand results in mutations at $\mathrm{dC}: \mathrm{dG}$ as well as $\mathrm{dA}$ :dT base pairs [57]. The repair of deaminated cytosines within switch regions, 5' of the constant regions of antibody genes, leads to the generation DSBs, which are a prerequisite 
for class switch recombination [59]. Hence, deficiency of functional AID in humans due to homozygous deletions or mutations causes the autosomal recessive form of an immunodeficiency syndrome, the hyperIgM syndrome [53]. Disruption of AID in the chicken B cell lymphoma cell line DT40 abolishes antibody diversification by gene conversion [60]. First studies suggested that the enzyme edits RNA intermediates [61], before it could be shown in vitro that AID deaminates ssDNA during transcription $[45,46,62]$, preferentially the $\mathrm{dC}$ within WRCY/RGYW motifs $[41,63]$.

Aside from mutational activity, cytidine deaminases have also been shown to participate in epigenetics through active DNA demethylation [64]. Cytosines in DNA can occur either in an unmethylated or methylated (5-methylcytosine) form and are often enriched in promoter regions in a $\mathrm{CpG}$ dinucleotide context where methylation is associated with gene silencing [64]. Deamination of methylated cytidines generates thymidines, which are removed by the DNA repair machinery [64]. Insertion of a non-methylated $\mathrm{dC}$ would hence result in $\mathrm{dC}$ demethylation [64]. While recent studies suggest that AID is involved in promoter demethylation during early embryonic development [6567], B cells from AID deficient mice did not exhibit any differences in methylated promoter regions [68].

\section{Regulation of AID activity}

While APOBEC proteins execute deamination without cofactors and are regulated primarily on the level of gene expression, AID is the only family member which is tightly regulated on several layers, apparently because AID is the only deaminase which targets genomic DNA as physiological substrate and hence, off-target DNA damage is likely to occur. These layers of regulation are I) transcription and alternative splicing, II) posttranslational modification, III) shuttling, and IV) interaction partners that specify targeting to certain DNA loci.

Basically, transcription of AID is induced in B cells in the context of the GC reaction after helper T cellmediated stimulation of B cells with CD40L and IL4 or TGF 3 [55], inducing the transcription factors $\mathrm{NFKB}$, Inhibitor of differentiation (Id) 2, Id3, the E-protein E47, PAX5, E2A and STAT6 [69-71]. Transcript levels are further regulated by alternative splicing, with only the full length AID variant being capable of mediating SHM and CSR [72-74]. As alternatively spliced AID transcripts are hardly detectable on protein level, alternative splicing is probably a further means to regulate the abundance of full length AID [75].

Concerning post translational modifications of AID, mass spectrometric and immunochemical approaches led to the identification of phosphorylation of several serine, threonine and tyrosine residues within the AID protein [76]. Of particular importance are amino acid residues S3 [77], S38 [76,78] and T140 [79], as their mutation to alanine does not impact catalytic activity, but interferes with CSR and SHM in vivo. This effect is particularly pronounced in AID haploinsufficient mice where AID levels are limited [80]. While phosphorylation of S38 was shown to be important for both processes, phosphorylation of T140 preferentially is necessary for somatic hypermutation of $\mathrm{V}$ region genes. In contrast, phosphorylation of S3 decreases AID activity [77]. In addition to phosphorylation, ubiquitination of AID was reported to occur thereby regulating protein abundance and activity [81,82]. AID continuously shuttles between cytoplasm and nucleus [83]. In particular, nuclear AID is efficiently polyubiquitinated followed by proteasomal degradation, while AID is relatively stable in the cytoplasm due to interaction with heat shock proteins [81,84-86]. Binding of nuclear AID to Reg- $\gamma$ also induces an ubiquitin-independent degradative process [87]. Hence, nuclear cytoplasmic shuttling is a further level of regulating AID activity and its access to DNA substrates. Several proteins have been shown to interact with AID in B cells. Replication protein A (RPA), which is associated with transcription bubbles and binds and stabilizes ssDNA, interacts with phosphorylated AID and most likely helps AID recruitment to transcribed Ig regions [88]. AID also associates with $\mathrm{UNG}$ and $\mathrm{MSH} 2 / 6$ heterodimers, which is important for initiation and resolution of DSBs at switch regions [89]. The protein kinase A (PKA) alpha regulatory subunit (PKAr1a) was found to be responsible for phosphorylation of S38 of AID on a consensus PKA site, allowing interaction with RPA and representing an event critical for CSR [76]. The ubiquitin ligase MDM2 interacts with the $\mathrm{C}$ terminus of AID but most likely does not significantly regulate AID activity in vivo [90]. CTNNBL1, a spliceosomal protein, is a further interaction partner of AID [91]. Although CTNNBL1 was shown to substantially regulate SHM in DT40 cells, it did not affect CSR in a mouse B cell line [92]. A protein known to regulate splicing, PTBP2, was identified to promote binding of AID to the transcribed switch region and significantly reduces class switch efficiency under knockdown conditions [93]. AID is also associated with stalled RNA polymerase II, and their binding is dependent on SPT5, just as the recruitment of AID to the switch region, as shown by quantitative PCRbased ChIP analysis [94]. Moreover, SPT5 colocalizes with AID and its occupancy is predictive for AID-induced mutations [94]. The switch region contains repetitive $5^{\circ}-$ AGCT-3' motifs that are bound by the 14-3-3 adapter protein, which is upregulated in B cells undergoing CSR [95]. Blocking of 14-3-3 impedes AID binding to the switch region and results in decreased CSR [95]. Another protein specifically expressed in GC B cells is GANP. It plays an important role in localizing AID in the nucleus and promotes binding of AID to the transcribed $\mathrm{IgV}$ region and therefore supports AID in SHM [96]. 
Targeting of AID is further regulated by cis-acting elements. Aside from transcription of target genes as prerequisite for AID to gain access to ssDNA within the transcription bubble [45], recent evidence reveals convergent sense and antisense transcription within topologically complexed DNA cluster to recruit AID mediated DNA deamination [97-99].

\section{AID and cancer}

A hallmark of B cell lymphomas is the occurrence of translocations between an oncogene and the Ig locus [100]. The most prominent example is the c-myc/ IgH translocation in Burkitt's lymphoma. By sequencing this particular translocation junction, it has long been known that c-myc is directly joined to the switch region and hence, a role for aberrant CSR in mediating this translocation was hypothesized [101]. Soon after AID has been discovered as key enzyme for SHM and CSR, researchers aimed at determining its contribution to translocations as a result of "mistargeted CSR". Indeed, subsequent studies revealed that c-myc/IgH translocations in IL6 transgenic mice are completely dependent on AID [102]. Moreover, by applying a deep-sequencing based method to document genome-wide chromosomal rearrangements in AID pro- and deficient mouse B cells, it became apparent that AID is responsible for translocations at multiple target genes, which are also found in human mature B cell lymphomas [103].

In addition to translocations, GC-derived lymphomas have been found to harbor many mutations in many non-Ig genes such as BCL6, CD79, CD95 and PAX5, which exhibit typical features of SHM [104-108]. Concomitantly, by comparing mutation frequencies in AID pro- versus deficient mice, it soon became clear that AID not only initiates the hypermutation of Ig genes but to a lesser extent also of many non-Ig genes, which are not properly protected by high-fidelity DNA repair, demonstrating off-target DNA damage induced by AID [109]. In addition, several reports show that many B cell lymphomas harbor subclonal heterogeneity at the Ig locus, which indicates ongoing AID activity during disease progression [110-113]. Hence, AID may not only contribute to malignant transformation but also to clonal evolution of B cell malignancies.

Aside from cancer of the B cell lineage, deregulated AID activity, mainly through overexpression, may induce various other types of cancer, such as gastric cancer $[114,115]$, liver cancer [116], breast and ovarian cancer [117], lung cancer and T cell lymphomas [118]. A study from Morisawa and co-workers on organ-specific genetic profiles from different tumors (liver, stomach, lung) in AID transgenic mice demonstrated that AID-mediated genetic changes were organ-specific and may thereby contribute to tissue-specific genetic diversity during cancer development [119]. An increased rate of TP53 mutations was found to be associated with an overexpression of AID mRNA in human lung cancer cell lines [120]. A recent study from Shimizu and colleagues on human and murine gastric tumors and tissues shows that somatic mutations are enriched in multiple genes in gastric mucosal tissues upon Helicobacter pylori infection and that increased AID activity in these tissues leads to the accumulation of these mutations, which may promote carcinogenesis in Helicobacter pylori infected patients [121]. Dysregulated AID expression can also be induced by inflammation and microbial infections, which then acts as a genotoxic factor in various human cancers [122]. Inflammatory signals that induce epithelial to mesenchymal transition are capable of inducing AID expression [123]. Recent work presents evidence that this inflammation-induced AID expression is required for epithelial to mesenchymal transition in ZR75.1 breast cancer cells and non-transformed mammary epithelial cells, since AID deficiency in these cells suppresses expression of key transcriptional regulators for epithelial to mesenchymal transition and is associated with increased cytosine methylation in promoters of these genes [123]. This study proposes, in accordance with other reports $[64,65,67]$, a role for AID in gene expression regulation and epigenetic reprogramming [123]. Furthermore, several oncogenic viruses can induce AID expression [124]. HCV, which is known to be a major cause of hepatocellular carcinoma, triggers AID expression in hepatocytes [116].

\section{APOBEC3 and cancer}

A study performed by Alexandrov et al. showed the first evidence that aside from AID, also other APOBEC family members could contribute to genomic DNA damage [38]. Whole exome sequencing (WES) and whole genome sequencing (WGS) approaches of more than seven thousand human cancers revealed the presence of an APOBEC mutation signature, i.e. $\mathrm{dC}>\mathrm{dT}$ transitions within a TCW (for APOBEC3) motif, in multiple cancer types, including bladder, breast, cervix and thyroid cancer, lung adenocarcinoma (LUAD), B cell lymphomas, multiple myelomas, acute lymphoblastic leukemia and chronic lymphocytic leukemia (CLL), whereas other cancers, like acute myeloid leukemia, liver and colorectal cancer, were devoid of such APOBEC signatures [38].

Moreover, a recent study from Burns and co-workers demonstrated that APOBEC3B mRNA is overexpressed in most primary breast tumors and breast cancer cell lines analyzed and that expression and activity of APOBEC3B correlated with genomic uracil levels, $\mathrm{dC}>\mathrm{dT}$ transition rates and mutation frequencies [37]. They confirmed this correlation through knockdown of endogenous APOBEC3B, which is predominantly localized in the nucleus and showed that the induction of APOBEC3B overexpression in vitro results in extensive DNA damage, cell cycle arrest and finally cell death [37]. Also copy 
number variations affecting the APOBEC3 gene cluster on chromosome 22 might be important in breast cancer, since a germline copy number polymorphism, in which the gene-coding region of $\mathrm{APOBEC} 3 \mathrm{~B}$ is deleted and its 3' UTR joined to APOBEC3A, associates with elevated risk of breast cancer $[125,126]$. This APOBEC3AAPOBEC3B germline polymorphism displays an elevated burden of APOBEC signature mutations, suggesting that this polymorphism presents cancer susceptibility through increased APOBEC activity [125]. Furthermore, APOBEC3B copy number alterations have been shown to be associated with decreased APOBEC3B expression in breast cancer cell lines [127]. When introducing APOBEC3A and APOBEC3B into yeast, genome-wide mutation patterns of APOBEC3A- and APOBEC3Bmediated deamination show strong similarity to mutation signatures found in breast cancer, which strengthens the proposed role of APOBEC3B, and maybe also APOBEC3A, in breast cancer hypermutation [35]. APOBEC3B was shown to be upregulated in several human lymphoma cell lines where cells with high APOBEC3B expression possessed mutations, mainly $\mathrm{dC}>\mathrm{dT}$ transitions, in actively transcribed oncogenes [128]. Also in CLL, APOBEC3B mutation signatures have been identified [129]. Overexpression of APOBEC3B in a APOBEC3B low expressing lymphoma cell line induced accumulation of $\mathrm{dC}>\mathrm{dT}$ mutation within the c-myc gene [128]. APOBEC3B is furthermore elevated in lung cancer, ovarian carcinoma, diverse ovarian cancer cell lines and high grade primary ovarian cancers [130,131]. WGS and expression profiling of 16 primary ovarian tumors revealed a correlation between APOBEC3B mRNA expression and total mutation load as well as transversion levels [130]. Due to the expression data and mutation

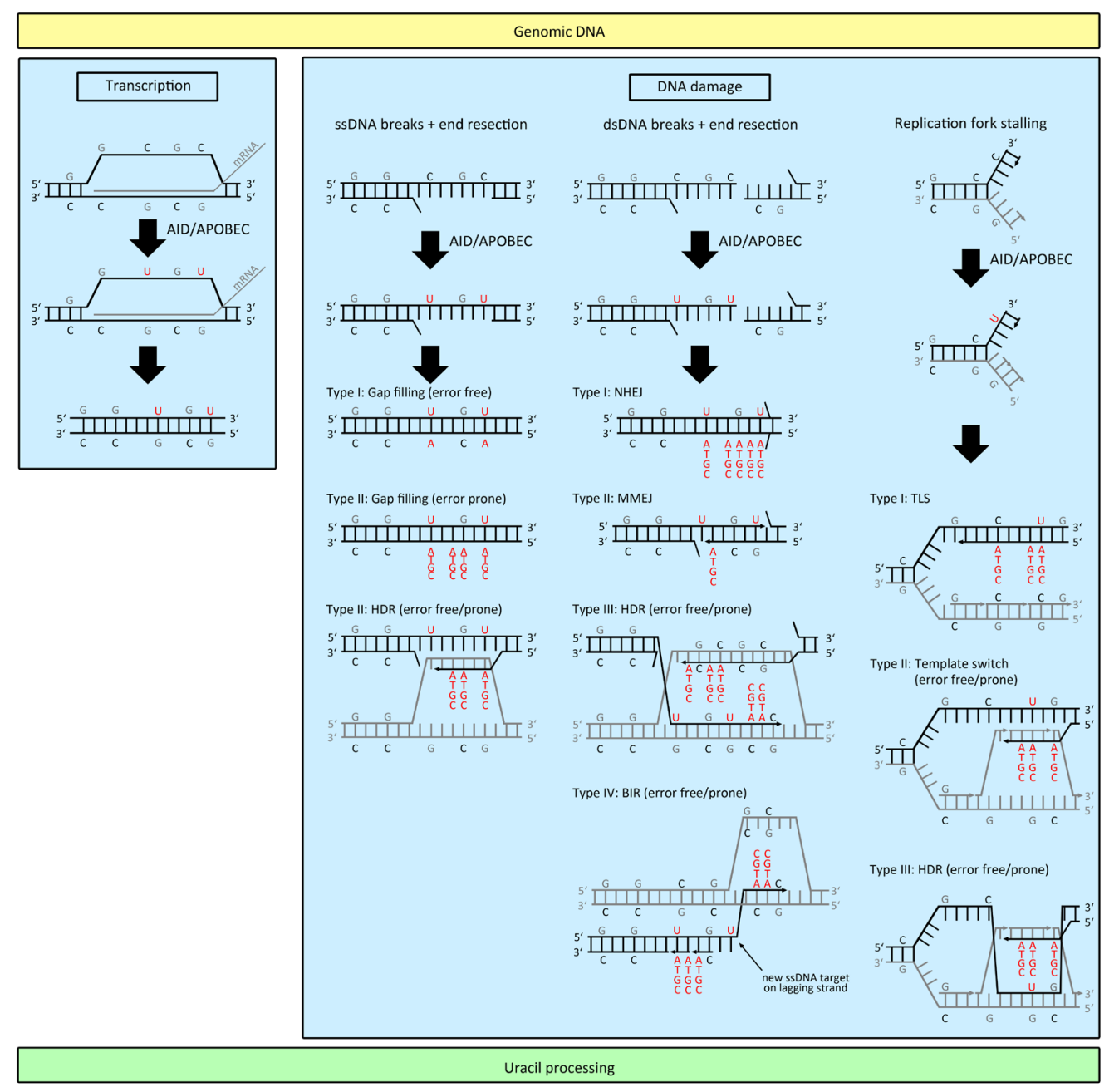

Figure 1: AID/APOBEC mediated mutagenesis of genomic DNA. Efficient dC deamination requires generation of ssDNA within genomic DNA. ssDNA can be generated by either transcription, DNA damage, which eventually leads to SSB and DSB, or by DNA replication in which replication fork stalling due to DNA lesions extends ssDNA formation. In either case, dUs generated on ssDNA remain in the dsDNA during error free or error prone repair of the initial lesion, leading to $\mathrm{dU} / \mathrm{dN}$ mismatches. (Abbreviations: SSB: single-strand break; DSB: double-strand break; HDR: homology-directed repair; NHEJ: non-homologous end joining; MMEJ: microhomology-mediated end joining; BIR: break-induced replication; TLS: translesion synthesis). 
patterns, it was suggested that APOBEC3B lesions are processed error prone and also attribute $\mathrm{APOBEC} 3 \mathrm{~B}$ a potential role in genomic instability in serous ovarian cancer [130]. It is also likely, that APOBEC 3 contributes to tumor progression in lung cancer, as a recent study demonstrated that a substantial amount of subclonal driver mutations occurred in an APOBEC sequence context [132]. Interestingly, increased APOBEC-associated mutation levels were found specifically in the region of LUAD, where the highest APOBEC3B mRNA levels were detected [132]. A cooperative role in APOBEC3B mutagenesis is furthermore attributed to the tumor suppressor fragile histidine triad protein (FHIT), which is known to cause DNA damage upon loss of protein activity in normal and cancer cells [133]. Frequencies of APOBEC3-mediated mutations were shown to vary upon changes in FHIT expression [133]. LUADs with low FHIT and high APOBEC3B expression harbored an increased number of APOBEC signature mutations, whereas samples with normal FHIT and high APOBEC3B expression did not show hypermutation due to APOBEC activity [133]. It was suggested that different levels of APOBEC3B and FHIT expression result in increased or decreased frequencies of APOBEC-mediated mutations [133].

Very recently, APOBEC3 proteins have been suggested to link viral infections to cancer development [134]. Stable transfection of normal breast epithelial cells with HPV was demonstrated to cause APOBEC3B mRNA overexpression leading to a significant increase in $\gamma$-H2AX foci formation and DNA breaks, which were blocked by knockdown of HPV and APOBEC3B [135]. For head and neck squamous cell carcinomas (HNSCCs) it has been shown by Henderson and colleagues that $\mathrm{HPV}^{+}$ compared to HPV tumors have elevated APOBEC3B and that PIK3CA helical domain mutations are APOBECdriven in multiple cancers, including $\mathrm{HPV}^{+} \mathrm{HNSCC}$ [136]. In addition, the authors describe a role for APOBECmediated mutagenesis in HPV-driven tumor development [136].

But also APOBEC 3 proteins aside from APOBEC 3B are reported to be implicated in cancer. APOBEC $3 \mathrm{G}$ was found to be highly expressed in colorectal tumors and hepatic metastasis and proposed to promote colorectal cancer hepatic metastasis through miR29 downregulation and consequent derepression of MMP2, a known metastasis activator [137]. Additionally, APOBEC3G acts as a prosurvival factor in lymphoma cells [51,52]. An inhibition of APOBEC $3 \mathrm{G}$ expression results in DSB repair deficiencies, which can be reconstituted by elevated APOBEC $3 \mathrm{G}$ levels, suggesting that APOBEC $3 \mathrm{G}$ recognizes dsDNA ends and facilitates rejoining through

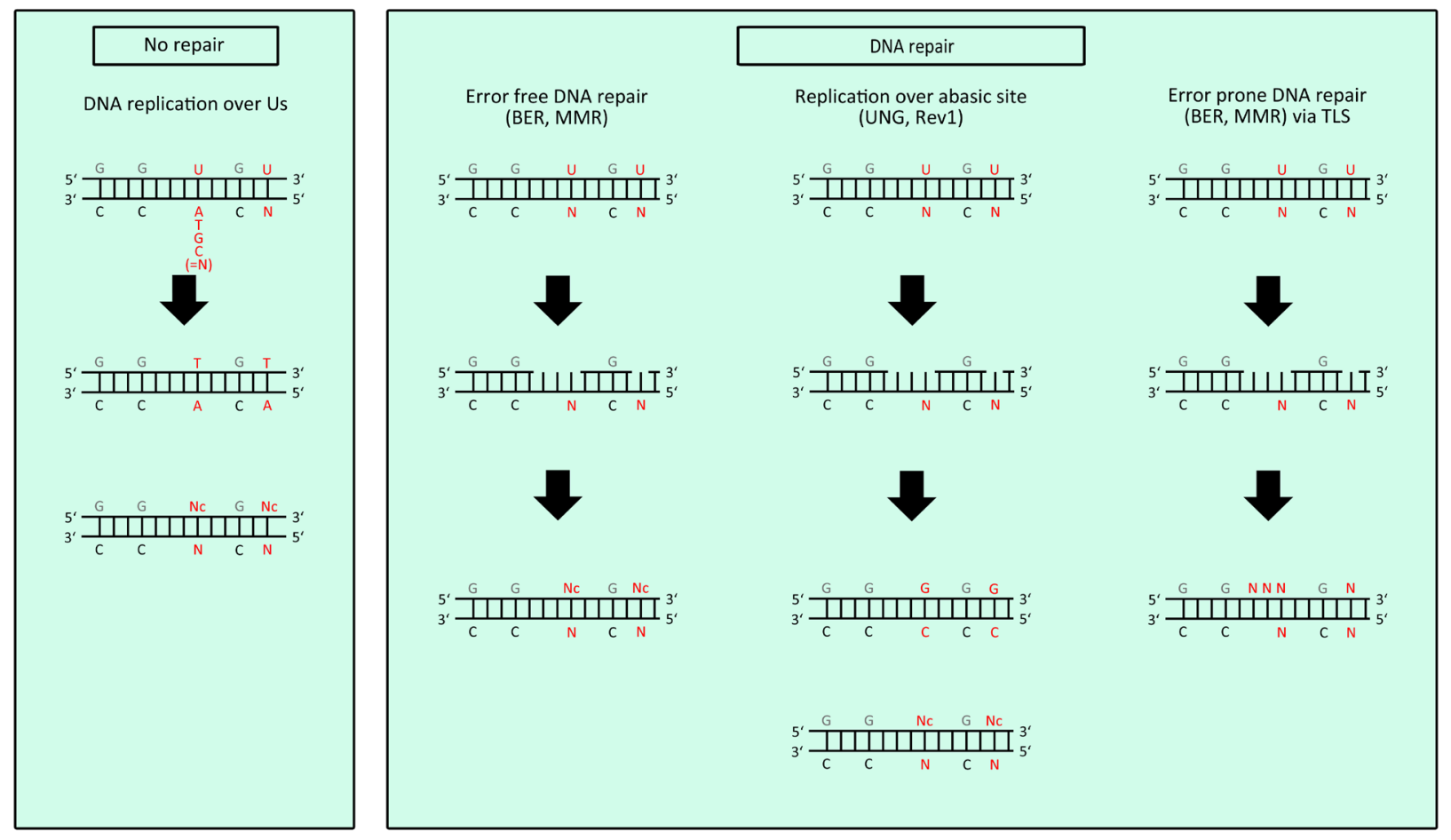

Figure 2: Processing of uracils within genomic DNA. dU/dN mismatches generated as described in Figure 1 are processed by different mechanisms: Replication of dU/dN mismatches eventually leads to dT/dA pairing (left panel). DNA repair (BER, MMR) can either occur error free or error prone, depending on which DNA polymerases are recruited. In case dUs are removed by UNG, replication over an abasic site often leads to the REV1 based incorporation of dCs opposite to the abasic site, which results in $\mathrm{dC}>\mathrm{dG}$ transversions. (Abbreviations: BER: base excision repair; MMR: mismatch repair; TLS: translesion synthesis; Nc: complementary base to N). 
NHEJ [51,52]. In a gene expression study comprising 67 diffuse large B cell lymphoma patients treated with R-CHOP (rituximab-cyclophosphamide, doxorubicin, vincristine, prednisone), a set of 16 genes, including APOBEC 3G, was identified to correlate with shortened overall survival and progression free survival [138]. Recent data link APOBEC3A activity to skin lesions, where APOBEC3-mediated transcription-dependent mutations, apart from CC dimer formation through UV radiation, are considered alternative initiating events in skin cancer [139]. Summarizing, APOBEC3 proteins are key players in cancer-associated somatic mutation processes and seem to influence cancer development and progression in several entities.

\section{Mutation clusters ('kataegis') in cancer}

An analysis of 21 breast cancer genomes uncovered the phenomenon of localized hypermutation, termed 'kataegis' (Greek for 'shower' or 'thunderstorm'), with mostly $\mathrm{dC}>\mathrm{dT}$ transitions at $\mathrm{TpC}$ dinucleotides, suggesting APOBEC3 proteins to be implicated in the formation of these mutation clusters [36]. Accumulated mutations have already been observed before, when Wang and colleagues sequenced the LacZ gene in spontaneous derived tumors from the Big Blue mouse and found multiple mutations with short intermutational distances [140]. Kataegis in many cancer types are associated with APOBEC3 enzymes, since mutation patterns are dominated by $\mathrm{dC}>\mathrm{dT}$ transitions and $\mathrm{dC}>\mathrm{dG}$ transversions, that occur within preferred APOBEC3 motifs [38]. APOBEC3B and $A P O B E C 3 A$ expression levels have been shown to correlate with kataegic mutation burden [141,142]. In vitro induction of mutation showers in yeast was observed upon expression of PmCDA1, a highly mutagenic cytosine deaminase from sea lamprey [143], or AID and APOBEC3B [35]. Although the deficiency of UNG dramatically increased the number of $\mathrm{dC}>\mathrm{dT}$ transitions in these experiments, the number of $\mathrm{dC}>\mathrm{dG}$ transversions as well as the amount of kataegic events was drastically reduced [35]. The same effect was observed in the absence of the error prone DNA polymerase REV1, which is able to insert nucleotides opposed to abasic sites with a strong preference for inserting $\mathrm{dCs}$, thus resulting in $\mathrm{dC}>\mathrm{dG}$ transversions [35].

Hence, a functional DNA repair machinery is a prerequisite for the induction of kataegis. The prevailing explanation for this observation is that DNA repair generates ssDNA substrates for APOBEC proteins during ssDNA resection (Figure 1). This means that DNA lesions either initiated through APOBEC enzymes themselves or by any other damaging agents (UV-light, radiation, ROS, etc.) induces DNA repair by either
BER or MMR, which comprise 5'-3' resection of the damaged DNA strand, leading to the generation of long ssDNA stretches that are attacked by APOBEC proteins. This repair-induced mutagenesis thus leads to strandcoordinated mutations, whereas only $\mathrm{dCs}$ on the nonresected DNA strand are deaminated, and to clusters of mutations, where mutations are focused on regions of a few kilobases, which are typically resected during DNA repair [144,145]. Interestingly, a portion of mutation clusters colocalizes with breakpoints of chromosomal rearrangements, DSBs or uncapped telomere ends $[35,36,146,147]$. During DSB repair, dsDNA ends can either be directly ligated by NHEJ or ssDNA is formed by 5'-3' end resection which facilitates homology-directed repair through microhomology-mediated end joining (MMEJ), homologous recombination or break-induced replication (BIR). In these cases, the generated ssDNA is again a substrate for APOBEC enzymes [35,36,146,147]. During BIR, a DSB is repaired by replication starting from the DSB using the homologous dsDNA strand as template $[148,149]$. As this repair pathway was reported to have a drastically delayed lagging strand compared to leading strand synthesis, ssDNA during lagging strand synthesis has a higher propensity to be attacked by APOBEC deaminases $[148,149]$. In this regard, any delay in lagging strand synthesis induced by e.g. polymerase stalling or interchain crosslink increases the likelihood that ssDNA within the replication fork is attacked by APOBEC proteins [148]. As a consequence of any attack by AID/ APOBEC members, dUs are accumulating in ssDNA, which possibly remain in dsDNA even after DNA repair (Figure 1). Replication over these dUs eventually results in $\mathrm{dC}>\mathrm{dT}$ mutations, whereas replication over abasic sites (in case $\mathrm{dU}$ is removed by UNG prior replication) is mainly accomplished by REV1, which preferentially incorporates $\mathrm{dCs}$ opposite to the abasic site, leading to $\mathrm{dC}>\mathrm{dG}$ transversions (Figure 2). Both mutations are typically enriched within kataegic events. Alternatively, dUs can be repaired by BER or MMR, where dUs are removed during 5'-3' resection of ssDNA batches followed by high fidelity gap filling by Pol $\delta$ or otherwise, by error prone gap filling by translesion synthesis (TLS) polymerases. The recruitment of error prone TLS polymerases is the main mechanism that provides AID-induced SHM of antibody variable genes [57] (Figure 2).

According to the literature it can be stated that the AID/APOBEC family of deaminases is likely to play an important role in mutagenesis and clonal evolution in cancer and moreover to be a key factor for kataegic events in cancer genomes. A major future challenge will be to assess whether presence of AID/APOBEC enzymes is sufficient to support mutagenesis or whether additional factors facilitate deaminase recruitment and accumulation of mutations. 


\section{ACKNOWLEDGMENTS}

This work was supported by a grant from the Austrian Science Fund FWF (P24619 to R.Ge.), the LIMCR-SCRI, and the province of Salzburg.

\section{CONFLICT OF INTEREST}

The authors declare no competing financial conflict of interest.

\section{REFERENCES}

1. Chen J, Miller BF, Furano AV. Repair of naturally occurring mismatches can induce mutations in flanking DNA. Elife. $2014 ; 3$.

2. Barnes DE and Lindahl T. Repair and genetic consequences of endogenous DNA base damage in mammalian cells. Annual Review of Genetics. 2004; 38: 445-476.

3. Lindahl T. Instability and Decay of the Primary Structure of Dna. Nature. 1993; 362: 709-715.

4. Neuberger MS, Harris RS, Di Noia J, Petersen-Mahrt SK. Immunity through DNA deamination. Trends in Biochemical Sciences. 2003; 28: 305-312.

5. Conticello SG, Thomas CJF, Petersen-Mahrt SK, Neuberger MS. Evolution of the AID/APOBEC family of polynucleotide (deoxy) cytidine deaminases. Molecular Biology and Evolution. 2005; 22: 367-377.

6. Harris RS and Liddament MT. Retroviral restriction by APOBEC proteins. Nature Reviews Immunology. 2004; 4: 868-877.

7. Muto T, Muramatsu M, Taniwaki M, Kinoshita K, Honjo T. Isolation, tissue distribution, and chromosomal localization of the human activation-induced cytidine deaminase (AID) gene. Genomics. 2000; 68: 85-88.

8. Espinosa R, Funahashi T, Hadjiagapiou C, Lebeau MM, Davidson NO. Assignment of the Gene Encoding the Human Apolipoprotein-B Messenger-Rna Editing Enzyme (Apobec1) to Chromosome 12P13.1. Genomics. 1994; 24: 414-415.

9. Liao W, Hong SH, Chan BHJ, Rudolph FB, Clark SC, Chan L. APOBEC-2, a cardiac- and skeletal muscle-specific member of the cytidine deaminase supergene family. Biochemical and Biophysical Research Communications. 1999; 260: 398-404.

10. Jarmuz A, Chester A, Bayliss J, Gisbourne J, Dunham I, Scott J, Navaratnam N. An anthropoid-specific locus of orphan C to U RNA-editing enzymes on chromosome 22. Genomics. 2002; 79: 285-296.

11. Rogozin IB, Basu MK, Jordan IK, Pavlov YI, Koonin EV. APOBEC4, a new member of the AID/APOBEC family of polynucleotide (deoxy) cytidine deaminases predicted by computational analysis. Cell Cycle. 2005; 4: 1281-1285.
12. Conticello SG. The AID/APOBEC family of nucleic acid mutators. Genome Biology. 2008; 9.

13. Munk C, Willemsen A, Bravo IG. An ancient history of gene duplications, fusions and losses in the evolution of APOBEC3 mutators in mammals. BMC Evolutionary Biology. 2012; 12: 71.

14. LaRue RS, Jonsson SR, Silverstein KA, Lajoie M, Bertrand D, El Mabrouk N, Hotzel I, Andresdottir V, Smith TP, Harris RS. The artiodactyl APOBEC3 innate immune repertoire shows evidence for a multi-functional domain organization that existed in the ancestor of placental mammals. BMC Molecular Biology. 2008; 9: 104.

15. Vieira VC and Soares MA. The Role of Cytidine Deaminases on Innate Immune Responses against Human Viral Infections. Biomed Research International. 2013.

16. Teng BB, Burant CF, Davidson NO. Molecular-Cloning of An Apolipoprotein-B Messenger-Rna Editing Protein. Science. 1993; 260: 1816-1819.

17. Navaratnam N, Morrison JR, Bhattacharya S, Patel D, Funahashi T, Giannoni F, Teng BB, Davidson NO, Scott J. The P27 Catalytic Subunit of the Apolipoprotein-B Messenger-Rna Editing Enzyme Is A Cytidine Deaminase. Journal of Biological Chemistry. 1993; 268: 20709-20712.

18. Chester A, Scott J, Anant S, Navaratnam N. RNA editing: cytidine to uridine conversion in apolipoprotein B mRNA. Biochimica et Biophysica Acta-Gene Structure and Expression. 2000; 1494: 1-13.

19. Rosenberg BR, Hamilton CE, Mwangi MM, Dewell S, Papavasiliou FN. Transcriptome-wide sequencing reveals numerous APOBEC1 mRNA-editing targets in transcript 3' UTRs. Nature Structural \& Molecular Biology. 2011; 18: 230-236

20. Anant $\mathrm{S}$ and Davidson NO. An AU-rich sequence element (UUUN[A/U] U) downstream of the edited $\mathrm{C}$ in apolipoprotein $\mathrm{B}$ mRNA is a high-affinity binding site for Apobec-1: binding of Apobec-1 to this motif in the 3' untranslated region of c-myc increases mRNA stability. Molecular and Cellular Biology. 2000; 20: 1982-1992.

21. Harris RS, Petersen-Mahrt SK, Neuberger MS. RNA editing enzyme APOBEC1 and some of its homologs can act as DNA mutators. Molecular Cell. 2002; 10: 1247-1253.

22. Lada AG, Krick CF, Kozmin SG, Mayorov VI, Karpova TS, Rogozin IB, Pavlov YI. Mutator effects and mutation signatures of editing deaminases produced in bacteria and yeast. Biochemistry. 2011; 76: 131-146.

23. Sato Y, Probst HC, Tatsumi R, Ikeuchi Y, Neuberger MS, Rada C. Deficiency in APOBEC2 Leads to a Shift in Muscle Fiber Type, Diminished Body Mass, and Myopathy. Journal of Biological Chemistry. 2010; 285: 7111-7118.

24. Sheehy AM, Gaddis NC, Choi JD, Malim MH. Isolation of a human gene that inhibits HIV-1 infection and is suppressed by the viral Vif protein. Nature. 2002; 418: 646650.

25. Bishop KN, Holmes RK, Sheehy AM, Davidson NO, 
Cho SJ, Malim MH. Cytidine deamination of retroviral DNA by diverse APOBEC proteins. Current Biology. 2004; 14: 1392-1396.

26. Liddament MT, Brown WL, Schumacher AJ, Harris RS. APOBEC3F properties and hypermutation preferences indicate activity against HIV-1 in vivo. Current Biology. 2004; 14: 1385-1391.

27. Zheng $\mathrm{YH}$, Irwin $\mathrm{D}$, Kurosu $\mathrm{T}$, Tokunaga $\mathrm{K}$, Sata $\mathrm{T}$, Peterlin BM. Human APOBEC3F is another host factor that blocks human immunodeficiency virus type 1 replication. Journal of Virology. 2004; 78: 6073-6076.

28. Wiegand HL, Doehle BP, Bogerd HP, Cullen BR. A second human antiretroviral factor, APOBEC 3F, is suppressed by the HIV-1 and HIV-2 Vif proteins. Embo Journal. 2004; 23: 2451-2458.

29. Chiu YL and Greene WC. The APOBEC3 cytidine deaminases: an innate defensive network opposing exogenous retroviruses and endogenous retroelements. Annual Review of Immunology. 2008; 26: 317-353.

30. Lackey L, Law EK, Brown WL, Harris RS. Subcellular localization of the APOBEC3 proteins during mitosis and implications for genomic DNA deamination. Cell Cycle. 2013; 12: 762-772.

31. Lackey L, Demorest ZL, Land AM, Hultquist JF, Brown WL, Harris RS. APOBEC3B and AID Have Similar Nuclear Import Mechanisms. Journal of Molecular Biology. 2012; 419: 301-314.

32. Kinomoto M, Kanno T, Shimura M, Ishizaka Y, Kojima A, Kurata T, Sata T, Tokunaga K. All APOBEC3 family proteins differentially inhibit LINE-1 retrotransposition. Nucleic Acids Research. 2007; 35: 2955-2964.

33. Li MMH and Emerman M. Polymorphism in Human APOBEC3H Affects a Phenotype Dominant for Subcellular Localization and Antiviral Activity. Journal of Virology. 2011; 85: 8197-8207.

34. Stenglein MD, Burns MB, Li M, Lengyel J, Harris RS. APOBEC3 proteins mediate the clearance of foreign DNA from human cells. Nature Structural \& Molecular Biology. 2010; 17: 222-U13.

35. Taylor BJ, Nik-Zainal S, Wu YL, Stebbings LA, Raine K, Campbell PJ, Rada C, Stratton MR, Neuberger MS. DNA deaminases induce break-associated mutation showers with implication of APOBEC3B and $3 \mathrm{~A}$ in breast cancer kataegis. Elife. 2013; 2: e00534.

36. Nik-Zainal S, Alexandrov LB, Wedge DC, Van Loo P, Greenman CD, Raine K, Jones D, Hinton J, Marshall J, Stebbings LA, Menzies A, Martin S, Leung K et al. Mutational Processes Molding the Genomes of 21 Breast Cancers. Cell. 2012; 149: 979-993.

37. Burns MB, Lackey L, Carpenter MA, Rathore A, Land AM, Leonard B, Refsland EW, Kotandeniya D, Tretyakova N, Nikas JB, Yee D, Temiz NIA, Donohue DE et al. APOBEC3B is an enzymatic source of mutation in breast cancer. Nature. 2013; 494: 366-370.
38. Alexandrov LB, Nik-Zainal S, Wedge DC, Aparicio SAJR, Behjati S, Biankin AV, Bignell GR, Bolli N, Borg A, Borresen-Dale AL, Boyault S, Burkhardt B, Butler AP et al. Signatures of mutational processes in human cancer. Nature. 2013; 500: 415-421.

39. Betz AG, Rada C, Pannell R, Milstein C, Neuberger MS. Passenger transgenes reveal intrinsic specificity of the antibody hypermutation mechanism: clustering, polarity, and specific hot spots. Proceedings of the National Academy of Sciences of the United States of America. 1993; 90: 2385-2388.

40. Rogozin IB and Kolchanov NA. Somatic Hypermutagenesis in Immunoglobulin Genes. 2. Influence of Neighboring Base Sequences on Mutagenesis. Biochimica et Biophysica Acta. 1992; 1171: 11-18.

41. Pham P, Bransteitter R, Petruska J, Goodman MF. Processive AID-catalysed cytosine deamination on singlestranded DNA simulates somatic hypermutation. Nature. 2003; 424: 103-107.

42. Refsland EW and Harris RS. The APOBEC3 family of retroelement restriction factors. Curr.Top.Microbiol. Immunol. 2013; 371: 1-27.

43. Beale RC, Petersen-Mahrt SK, Watt IN, Harris RS, Rada C, Neuberger MS. Comparison of the differential contextdependence of DNA deamination by APOBEC enzymes: correlation with mutation spectra in vivo. Journal of Molecular Biology. 2004; 337: 585-596.

44. Smith HC, Bennett RP, Kizilyer A, McDougall WM, Prohaska KM. Functions and regulation of the APOBEC family of proteins. Seminars in Cell \& Developmental Biology. 2012; 23: 258-268.

45. Petersen-Mahrt SK, Harris RS, Neuberger MS. AID mutates E-coli suggesting a DNA deamination mechanism for antibody diversification. Nature. 2002; 418: 99-103.

46. Chaudhuri J, Tian M, Khuong C, Chua K, Pinaud E, Alt FW. Transcription-targeted DNA deamination by the AID antibody diversification enzyme. Nature. 2003; 422: 726-730.

47. Bransteitter R, Pham P, Scharff MD, Goodman MF. Activation-induced cytidine deaminase deaminates deoxycytidine on single-stranded DNA but requires the action of RNase. Proceedings of the National Academy of Sciences of the United States of America. 2003; 100: 4102-4107.

48. Refsland EW, Stenglein MD, Shindo K, Albin JS, Brown WL, Harris RS. Quantitative profiling of the full APOBEC3 mRNA repertoire in lymphocytes and tissues: implications for HIV-1 restriction. Nucleic Acids Research. 2010; 38 : 4274-4284.

49. Mikl MC, Watt IN, Lu M, Reik W, Davies SL, Neuberger MS, Rada C. Mice deficient in APOBEC2 and APOBEC3. Molecular and Cell Biology. 2005; 25: 72707277.

50. Halemano K, Guo KJ, Heilman KJ, Barrett BS, Smith DS, 
Hasenkrug KJ, Santiago ML. Immunoglobulin somatic hypermutation by APOBEC3/Rfv3 during retroviral infection. Proceedings of the National Academy of Sciences of the United States of America. 2014; 111: 7759-7764.

51. Nowarski R, Wilner OI, Cheshin O, Shahar OD, Kenig E, Baraz L, Britan-Rosich E, Nagler A, Harris RS, Goldberg M, Willner I, Kotler M. APOBEC3G enhances lymphoma cell radioresistance by promoting cytidine deaminasedependent DNA repair. Blood. 2012; 120: 366-375.

52. Nowarski R and Kotler M. APOBEC3 Cytidine Deaminases in Double-Strand DNA Break Repair and Cancer Promotion. Cancer Research. 2013; 73: 3494-3498.

53. Revy P, Muto T, Levy Y, Geissmann F, Plebani A, Sanal O, Catalan N, Forveille M, Dufourcq-Lagelouse R, Gennery A, Tezcan I, Ersoy F, Kayserili H et al. Activation-induced cytidine deaminase (AID) deficiency causes the autosomal recessive form of the hyper-IgM syndrome (HIGM2). Cell. 2000; 102: 565-575.

54. Muramatsu M, Sankaranand VS, Anant S, Sugai M, Kinoshita K, Davidson NO, Honjo T. Specific expression of activation-induced cytidine deaminase (AID), a novel member of the RNA-editing deaminase family in germinal center B cells. Journal of Biological Chemistry. 1999; 274: 18470-18476.

55. Muramatsu M, Kinoshita K, Fagarasan S, Yamada S, Shinkai Y, Honjo T. Class switch recombination and hypermutation require activation-induced cytidine deaminase (AID), a potential RNA editing enzyme. Cell. 2000; 102: 553-563.

56. Conticello SG. Creative deaminases, self-inflicted damage, and genome evolution. Effects of Genome Structure and Sequence on Variation and Evolution. 2012; 1267: 79-85.

57. Di Nola JM and Neuberger MS. Molecular mechanisms of antibody somatic hypermutation. Annual Review of Biochemistry. 2007; 76: 1-22.

58. Chaudhuri $\mathrm{J}$ and Alt FW. Class-switch recombination: Interplay of transcription, DNA deamination and DNA repair. Nature Reviews Immunology. 2004; 4: 541-552.

59. Xu ZM, Zan H, Pone EJ, Mai T, Casali P. Immunoglobulin class-switch DNA recombination: induction, targeting and beyond. Nature Reviews Immunology. 2012; 12: 517-531.

60. Harris RS, Sale JE, Petersen-Mahrt SK, Neuberger MS. AID is essential for immunoglobulin $\mathrm{V}$ gene conversion in a cultured B cell line. Current Biology. 2002; 12: 435-438.

61. Doi T, Kinoshita K, Ikegawa M, Muramatsu M, Honjo T. De novo protein synthesis is required for the activation-induced cytidine deaminase function in class-switch recombination. Proceedings of the National Academy of Sciences of the United States of America. 2003; 100: 2634-2638.

62. Rada C, Williams GT, Nilsen H, Barnes DE, Lindahl T, Neuberger MS. Immunoglobulin isotype switching is inhibited and somatic hypermutation perturbed in UNGdeficient mice. Current Biology. 2002; 12: 1748-1755.

63. Rogozin IB and Kolchanov NA. Somatic Hypermutagenesis in Immunoglobulin Genes.2. Influence of Neighboring Base Sequences on Mutagenesis. Biochimica et Biophysica Acta. 1992; 1171: 11-18.

64. Teperek-Tkacz M, Pasque V, Gentsch G, FergusonSmith AC. Epigenetic reprogramming: is deamination key to active DNA demethylation? Reproduction. 2011; 142: 621-632.

65. Bhutani N, Brady JJ, Damian M, Sacco A, Corbel SY, Blau HM. Reprogramming towards pluripotency requires AID-dependent DNA demethylation. Nature. 2010; 463: 1042-1U57.

66. Rai K, Huggins IJ, James SR, Karpf AR, Jones DA, Cairns BR. DNA Demethylation in Zebrafish Involves the Coupling of a Deaminase, a Glycosylase, and Gadd45. Cell. 2008; 135: 1201-1212.

67. Popp C, Dean W, Feng SH, Cokus SJ, Andrews S, Pellegrini M, Jacobsen SE, Reik W. Genome-wide erasure of DNA methylation in mouse primordial germ cells is affected by AID deficiency. Nature. 2010; 463: 1101-U126.

68. Fritz EL, Rosenberg BR, Lay K, Mihailovic A, Tuschl T, Papavasiliou FN. A comprehensive analysis of the effects of the deaminase AID on the transcriptome and methylome of activated B cells. Nature Immunology. 2013; 14: 749-755.

69. Sayegh CE, Quong MW, Agata Y, Murre C. E-proteins directly regulate expression of activation-induced deaminase in mature B cells. Nature Immunology. 2003; 4: 586-593.

70. Dedeoglu F, Horwitz B, Chaudhuri J, Alt FW, Geha RS. Induction of activation-induced cytidine deaminase gene expression by IL-4 and CD40 ligation is dependent on STAT6 and NF kappa B. International Immunology. 2004; 16: 395-404.

71. Gonda H, Sugai M, Nambu Y, Katakai T, Agata Y, Mori KJ, Yokota Y, Shimizu A. The balance between Pax5 and Id2 activities is the key to AID gene expression. Journal of Experimental Medicine. 2003; 198: 1427-1437.

72. Wu XS, Darce JR, Chang SK, Nowakowski GS, Jelinek DF. Alternative splicing regulates activation-induced cytidine deaminase (AID): implications for suppression of AID mutagenic activity in normal and malignant B cells. Blood. 2008; 112: 4675-4682.

73. van Maldegem F, Scheeren FA, Jibodh RA, Bende RJ, Jacobs H, van Noesel CJM. AID splice variants lack deaminase activity. Blood. 2009; 113: 1862-1864.

74. van Maldegem F, Jibodh RA, van Dijk R, Bende RJ, van Noesel CJM. Activation-Induced Cytidine Deaminase Splice Variants Are Defective Because of the Lack of Structural Support for the Catalytic Site. Journal of Immunology. 2010; 184: 2487-2491.

75. Rebhandl S, Huemer M, Zaborsky N, Gassner FJ, Catakovic K, Felder TK, Greil R, Geisberger R. Alternative splice variants of AID are not stoichiometrically present at the protein level in chronic lymphocytic leukemia. European Journal of Immunology. 2014; 44: 2175-2187. 
76. Basu U, Chaudhuri J, Alpert C, Dutt S, Ranganath S, Li G, Schrum JP, Manis JP, Alt FW. The AID antibody diversification enzyme is regulated by protein kinase A phosphorylation. Nature. 2005; 438: 508-511.

77. Gazumyan A, Timachova K, Yuen G, Siden E, Di Virgilio M, Woo EM, Chait BT, San Martin BR, Nussenzweig MC, McBride KM. Amino-Terminal Phosphorylation of Activation-Induced Cytidine Deaminase Suppresses c-myc/IgH Translocation. Molecular and Cellular Biology. 2011; 31: 442-449.

78. McBride KM, Gazumyan A, Woo EM, Barreto VM, Robbiani DF, Chait BT, Nussenzweig MC. Regulation of hypermutation by activation-induced cytidine dearninase phosphorylation. Proceedings of the National Academy of Sciences of the United States of America. 2006; 103: 8798-8803.

79. McBride KM, Gazumyan A, Woo EM, Schwickert TA, Chait BT, Nussenzweig MC. Regulation of class switch recombination and somatic mutation by AID phosphorylation. Journal of Experimental Medicine. 2008; 205: 2585-2594.

80. Sernandez IV, de Yebenes VG, Dorsett Y, Ramiro AR. Haploinsufficiency of Activation-Induced Deaminase for Antibody Diversification and Chromosome Translocations both In Vitro and In Vivo. PLoS One. 2008; 3.

81. Aoufouchi S, Faili A, Zober C, D’Orlando O, Weller S, Weill JC, Reynaud CA. Proteasomal degradation restricts the nuclear lifespan of AID. Journal of Experimental Medicine. 2008; 205: 1357-1368.

82. Geisberger R, Huemer M, Gassner FJ, Zaborsky N, Egle A, Greil R. Lysine residue at position 22 of the AID protein regulates its class switch activity. PLoS One. 2012; 7: e30667.

83. Ito S, Nagaoka H, Shinkura R, Begum N, Muramatsu M, Nakata M, Honjo T. Activation-induced cytidine deaminase shuttles between nucleus and cytoplasm like apolipoprotein B mRNA editing catalytic polypeptide 1. Proceedings of the National Academy of Sciences of the United States of America. 2004; 101: 1975-1980.

84. Geisberger R, Rada C, Neuberger MS. The stability of AID and its function in class-switching are critically sensitive to the identity of its nuclear-export sequence. Proceedings of the National Academy of Sciences of the United States of America. 2009; 106: 6736-6741.

85. Orthwein A, Patenaude AM, Affar E, Lamarre A, Young JC, Di Noia JM. Regulation of activation-induced deaminase stability and antibody gene diversification by Hsp90. Journal of Experimental Medicine. 2010; 207: 2751-2765.

86. Orthwein A, Zahn A, Methot SP, Godin D, Conticello SG, Terada K, Di Noia JM. Optimal functional levels of activation-induced deaminase specifically require the Hsp40 DnaJa1. Embo Journal. 2012; 31: 679-691.

87. Uchimura Y, Barton LF, Rada C, Neuberger MS. REGgamma associates with and modulates the abundance of nuclear activation-induced deaminase. Journal of
Experimental Medicine. 2011; 208: 2385-2391.

88. Chaudhuri J, Khuong C, Alt FW. Replication protein A interacts with AID to promote deamination of somatic hypermutation targets. Nature. 2004; 430: 992-998.

89. Ranjit S, Khair L, Linehan EK, Ucher AJ, Chakrabarti M, Schrader CE, Stavnezer J. AID recruits UNG and Msh2 to Ig switch regions dependent upon the AID C terminus [corrected]. Journal of Immunology. 2011; 187: 2464-2475.

90. MacDuff DA, Neuberger MS, Harris RS. MDM2 can interact with the C-terminus of AID but it is inessential for antibody diversification in DT40 B cells. Molecular Immunology. 2006; 43: 1099-1108.

91. Conticello SG, Ganesh K, Xue K, Lu M, Rada C, Neuberger MS. Interaction between anti bodydiversification enzyme AID and spliceosome-associated factor CTNNBL1. Molecular Cell. 2008; 31: 474-484.

92. Han L, Masani S, Yu K. Cutting edge: CTNNBL1 is dispensable for Ig class switch recombination. Journal of Immunology. 2010; 185: 1379-1381.

93. Nowak U, Matthews AJ, Zheng SM, Chaudhuri J. The splicing regulator PTBP2 interacts with the cytidine deaminase AID and promotes binding of AID to switchregion DNA. Nature Immunology. 2011; 12: 160-U73.

94. Pavri R, Gazumyan A, Jankovic M, Di Virgilio M, Klein I, Ansarah-Sobrinho C, Resch W, Yamane A, San Martin BR, Barreto V, Nieland TJ, Root DE, Casellas R et al. Activation-Induced Cytidine Deaminase Targets DNA at Sites of RNA Polymerase II Stalling by Interaction with Spt5. Cell. 2010; 143: 122-133.

95. Xu ZM, Fulop Z, Wu GK, Pone EJ, Zhang JS, Mai T, Thomas LM, Al Qahtani A, White CA, Park SR, Steinacker P, Li ZG, Yates J et al. 14-3-3 adaptor proteins recruit AID to 5 '-AGCT-3 '-rich switch regions for class switch recombination. Nature Structural \& Molecular Biology. 2010; 17: 1124-1U13.

96. Maeda K, Singh SK, Eda K, Kitabatake M, Pham P, Goodman MF, Sakaguchi N. GANP-mediated Recruitment of Activation-induced Cytidine Deaminase to Cell Nuclei and to Immunoglobulin Variable Region DNA. Journal of Biological Chemistry. 2010; 285: 23945-23953.

97. Pefanis E, Wang J, Rothschild G, Lim J, Chao J, Rabadan R, Economides AN, Basu U. Noncoding RNA transcription targets AID to divergently transcribed loci in B cells. Nature. 2014; 514: 389-393.

98. Meng FL, Du Z, Federation A, Hu J, Wang Q, KiefferKwon KR, Meyers RM, Amor C, Wasserman CR, Neuberg D, Casellas R, Nussenzweig MC, Bradner JE et al. Convergent Transcription at Intragenic Super-Enhancers Targets AID-Initiated Genomic Instability. Cell. 2014.

99. Qian J, Wang Q, Dose M, Pruett N, Kieffer-Kwon KR, Resch W, Liang G, Tang Z, Mathe E, Benner C, Dubois W, Nelson S, Vian L et al. B Cell Super-Enhancers and Regulatory Clusters Recruit AID Tumorigenic Activity. Cell. 2014. 
100. Kuppers R. Mechanisms of B-cell lymphoma pathogenesis. Nature Reviews Cancer. 2005; 5: 251-262.

101. Adams JM, Gerondakis S, Webb E, Corcoran LM, Cory S. Cellular myc oncogene is altered by chromosome translocation to an immunoglobulin locus in murine plasmacytomas and is rearranged similarly in human Burkitt lymphomas. Proceedings of the National Academy of Sciences of the United States of America. 1983; 80: 1982-1986.

102. Ramiro AR, Jankovic M, Eisenreich T, Difilippantonio S, Chen-Kiang S, Muramatsu M, Hongo T, Nussenzweig A, Nussenzweig MC. AID is required for c-myc/IgH chromosome translocations in vivo. Cell. 2004; 118: 431-438.

103. Klein IA, Resch W, Jankovic M, Oliveira T, Yamane A, Nakahashi H, Di Virgilio M, Bothmer A, Nussenzweig A, Robbiani DF, Casellas R, Nussenzweig MC. TranslocationCapture Sequencing Reveals the Extent and Nature of Chromosomal Rearrangements in B Lymphocytes. Cell. 2011; 147: 95-106.

104. Shen HM, Peters A, Baron B, Zhu XD, Storb U. Mutation of BCL- 6 gene in normal B cells by the process of somatic hypermutation of Ig genes. Science. 1998; 280: 1750-1752.

105. Pasqualucci L, Migliazza A, Fracchiolla N, William C, Neri A, Baldini L, Chaganti RSK, Klein U, Kuppers R, Rajewsky K, Dalla-Favera R. BCL-6 mutations in normal germinal center B cells: Evidence of somatic hypermutation acting outside Ig loci. Proceedings of the National Academy of Sciences of the United States of America. 1998; 95: 11816-11821.

106. Gordon MS, Kanegai CM, Doerr JR, Wall R. Somatic hypermutation of the B cell receptor genes B29 (Ig beta, CD79b) and mb1 (Ig alpha, CD79a). Proceedings of the National Academy of Sciences of the United States of America. 2003; 100: 4126-4131.

107. Muschen M, Re D, Jungnickel B, Diehl V, Rajewsky K, Kuppers R. Somatic mutation of the CD95 gene in human B cells as a side-effect of the germinal center reaction. Journal of Experimental Medicine. 2000; 192: 1833-1839.

108. Pasqualucci L, Bhagat G, Jankovic M, Compagno M, Smith P, Muramatsu M, Honjo T, Morse HC, Nussenzweig MC, Dalla-Favera R. AID is required for germinal center-derived lymphomagenesis. Nature Genetics. 2008; 40: 108-112.

109. Liu M, Duke JL, Richter DJ, Vinuesa CG, Goodnow CC, Kleinstein SH, Schatz DG. Two levels of protection for the B cell genome during somatic hypermutation. Nature. 2008; 451: 841-U11.

110. Huemer M, Rebhandl S, Zaborsky N, Gassner FJ, Hainzl S, Weiss L, Hebenstreit D, Greil R, Geisberger R. AID induces intraclonal diversity and genomic damage in CD86 chronic lymphocytic leukemia cells. European Journal of Immunology. 2014.

111. Ottensmeier CH, Thompsett AR, Zhu DL, Wilkins BS,
Sweetenham JW, Stevenson FK. Analysis of V-H genes in follicular and diffuse lymphoma shows ongoing somatic mutation and multiple isotype transcripts in early disease with changes during disease progression. Blood. 1998; 91: 4292-4299.

112. Hardianti MS, Tatsumi E, Syampurnawati M, Furuta K, Saigo K, Nakamachi Y, Kumagai S, Ohno H, Tanabe S, Uchida M, Yasuda N. Activation-induced cytidine deaminase expression in follicular lymphoma: association between AID expression and ongoing mutation in FL. Leukemia. 2004; 18: 826-831.

113. Campbell PJ, Pleasance ED, Stephens PJ, Dicks E, Rance R, Goodhead I, Follows GA, Green AR, Futreal PA, Stratton MR. Subclonal phylogenetic structures in cancer revealed by ultra-deep sequencing. Proceedings of the National Academy of Sciences of the United States of America. 2008; 105: 13081-13086.

114. Matsumoto Y, Marusawa H, Kinoshita K, Endo Y, Kou T, Morisawa T, Azuma T, Okazaki IM, Honjo T, Chiba T. Helicobacter pylori infection triggers aberrant expression of activation-induced cytidine deaminase in gastric epithelium. Nature Medicine. 2007; 13: 470-476.

115. Matsumoto Y, Marusawa H, Kinoshita K, Niwa Y, Sakai Y, Chiba T. Up-regulation of Activation-Induced Cytidine Deaminase Causes Genetic Aberrations at the CDKN2bCDKN2a in Gastric Cancer. Gastroenterology. 2010; 139: 1984-1994.

116. Endo Y, Marusawa H, Kinoshita K, Morisawa T, Sakurai T, Okazaki IM, Watashi K, Shimotohno K, Honjo T, Chiba T. Expression of activation-induced cytidine deaminase in human hepatocytes via NF-kappa B signaling. Oncogene. 2007; 26: 5587-5595.

117. Pauklin S, Scrnandez IV, Bachmann G, Ramiro AR, Petersen-Mahrt SK. Estrogen directly activates AID transcription and function. Journal of Experimental Medicine. 2009; 206: 99-111.

118. Okazaki I, Hiai H, Kakazu N, Yamada S, Muramatsu M, Kinoshita K, Honjo T. Constitutive expression of AID leads to tumorigenesis. Journal of Experimental Medicine. 2003; 197: 1173-1181.

119. Morisawa T, Marusawa H, Ueda Y, Iwai A, Okazaki I, Honjo T, Chiba T. Organ-specific profiles of genetic changes in cancers caused by activation-induced cytidine deaminase expression. International Journal of Cancer. 2008; 123: 2735-2740.

120. Shinmura $\mathrm{K}$, Igarashi $\mathrm{H}$, Goto $\mathrm{M}$, Tao $\mathrm{H}$, Yamada $\mathrm{H}$, Matsuura S, Tajima M, Matsuda T, Yamane A, Funai K, Tanahashi M, Niwa H, Ogawa $\mathrm{H}$ et al. Aberrant Expression and Mutation-Inducing Activity of AID in Human Lung Cancer. Annals of Surgical Oncology. 2011; 18: 2084-2092.

121. Shimizu T, Marusawa H, Matsumoto Y, Inuzuka T, Ikeda A, Fujii Y, Minamiguchi S, Miyamoto S, Kou T, Sakai Y, Crabtree JE, Chiba T. Accumulation of Somatic Mutations in TP53 in Gastric Epithelium With Helicobacter pylori Infection. Gastroenterology. 2014; 147: 407-417. 
122. Park SR. Activation-induced Cytidine Deaminase in B Cell Immunity and Cancers. Immune Network. 2012; 12: 230-239.

123. Munoz DP, Lee EL, Takayama S, Coppe JP, Heo SJ, Boffelli D, Di Noia JM, Martin DIK. Activation-induced cytidine deaminase (AID) is necessary for the epithelialmesenchymal transition in mammary epithelial cells. Proceedings of the National Academy of Sciences of the United States of America. 2013; 110: E2977-E2986.

124. Mechtcheriakova D, Svoboda M, Meshcheryakova A, Jensen-Jarolim E. Activation-induced cytidine deaminase (AID) linking immunity, chronic inflammation, and cancer. Cancer Immunology Immunotherapy. 2012; 61: 1591-1598.

125. Nik-Zainal S, Wedge DC, Alexandrov LB, Petljak M, Butler AP, Bolli N, Davies HR, Knappskog S, Martin S, Papaemmanuil E, Ramakrishna M, Shlien A, Simonic I et al. Association of a germline copy number polymorphism of APOBEC3A and APOBEC3B with burden of putative APOBEC-dependent mutations in breast cancer. Nature Genetics. 2014; 46: 487-491.

126. Long J, Delahanty RJ, Li G, Gao YT, Lu W, Cai Q, Xiang YB, Li C, Ji BT, Zheng Y, Ali S, Shu XO, Zheng W. A common deletion in the APOBEC3 genes and breast cancer risk. Journal of the National Cancer Institute. 2013; 105: 573-579.

127. Komatsu A, Nagasaki K, Fujimori M, Amano J, Miki Y. Identification of novel deletion polymorphisms in breast cancer. International Journal of Oncology. 2008; 33: 261-270.

128. Shinohara M, Io K, Shindo K, Matsui M, Sakamoto T, Tada K, Kobayashi M, Kadowaki N, Takaori-Kondo A. APOBEC3B can impair genomic stability by inducing base substitutions in genomic DNA in human cells. Scientific Reports. 2012; 2.

129. Rebhandl S, Huemer M, Gassner FJ, Zaborsky N, Hebenstreit D, Catakovic K, Grossinger EM, Greil R, Geisberger R. APOBEC3 signature mutations in chronic lymphocytic leukemia. Leukemia. 2014; 28: 1929-1932.

130. Leonard B, Hart SN, Burns MB, Carpenter MA, Temiz NA, Rathore A, Vogel RI, Nikas JB, Law EK, Brown WL, Li Y, Zhang YJ, Maurer MJ et al. APOBEC3B Upregulation and Genomic Mutation Patterns in Serous Ovarian Carcinoma. Cancer Research. 2013; 73: 7222-7231.

131. Sasaki H, Suzuki A, Tatematsu T, Shitara M, Hikosaka Y, Okuda K, Moriyama S, Yano M, Fujii Y. APOBEC3B gene overexpression in non-small-cell lung cancer. Biomed Reports. 2014; 2: 392-395.

132. de Bruin EC, McGranahan N, Mitter R, Salm M, Wedge DC, Yates L, Jamal-Hanjani M, Shafi S, Murugaesu N, Rowan AJ, Gronroos E, Muhammad MA, Horswell S et al. Spatial and temporal diversity in genomic instability processes defines lung cancer evolution. Science. 2014; 346: 251-256.

133. Waters CE, Saldivar JC, Amin ZA, Schrock MS, Huebner
K. FHIT loss-induced DNA damage creates optimal APOBEC substrates: Insights into APOBEC-mediated mutagenesis. Oncotarget. 2015; 6(4): 3409-3419.

134. Downey RF, Sullivan FJ, Wang-Johanning F, Ambs S, Giles FJ, Glynn SA. Human endogenous retrovirus K and cancer: Innocent bystander or tumorigenic accomplice? International Journal of Cancer. 2014.

135. Ohba K, Ichiyama K, Yajima M, Gemma N, Nikaido M, Wu QQ, Chong PP, Mori S, Yamamoto R, Wong JEL, Yamamoto N. In Vivo and In Vitro Studies Suggest a Possible Involvement of HPV Infection in the Early Stage of Breast Carcinogenesis via APOBEC3B Induction. PLoS One. 2014; 9.

136. Henderson S, Chakravarthy A, Su X, Boshoff C, Fenton TR. APOBEC-mediated cytosine deamination links PIK3CA helical domain mutations to human papillomavirus-driven tumor development. Cell Reports. 2014; 7: 1833-1841.

137. Ding QQ, Chang CJ, Xie XM, Xia WY, Yang JY, Wang SC, Wang Y, Xia JH, Chen LB, Cai CC, Li HB, Yen CJ, Kuo HP et al. APOBEC3G promotes liver metastasis in an orthotopic mouse model of colorectal cancer and predicts human hepatic metastasis. Journal of Clinical Investigation. 2011; 121: 4526-4536.

138. Jais JP, Haioun C, Molina TJ, Rickman DS, de Reynies A, Berger F, Gisselbrecht C, Briere J, Reyes F, Gaulard P, Feugier P, Labouyrie E, Tilly $\mathrm{H}$ et al. The expression of 16 genes related to the cell of origin and immune response predicts survival in elderly patients with diffuse large B-cell lymphoma treated with $\mathrm{CHOP}$ and rituximab. Leukemia. 2008; 22: 1917-1924.

139. Pham P, Landolph A, Mendez C, Li N, Goodman MF. A Biochemical Analysis Linking APOBEC3A to Disparate HIV-1 Restriction and Skin Cancer. Journal of Biological Chemistry. 2013; 288: 29294-29304.

140. Wang J, Gonzalez KD, Scaringe WA, Tsai K, Liu N, Gu D, Li W, Hill KA, Sommer SS. Evidence for mutation showers. Proceedings of the National Academy of Sciences of the United States of America. 2007; 104: 8403-8408.

141. Jia P, Pao W, Zhao Z. Patterns and processes of somatic mutations in nine major cancers. BMC Medical Genomics. 2014; 7: 11.

142. Roberts SA, Lawrence MS, Klimczak LJ, Grimm SA, Fargo D, Stojanov P, Kiezun A, Kryukov GV, Carter SL, Saksena G, Harris S, Shah RR, Resnick MA et al. An APOBEC cytidine deaminase mutagenesis pattern is widespread in human cancers. Nature Genetics. 2013; 45: 970-+.

143. Lada AG, Dhar A, Boissy RJ, Hirano M, Rubel AA, Rogozin IB, Pavlov YI. AID/APOBEC cytosine deaminase induces genome-wide kataegis. Biology Direct. 2012; 7: 47.

144. Chen J, Miller BF, Furano AV. Repair of naturally occurring mismatches can induce mutations in flanking DNA. Elife. $2014 ; 3$.

145. Wilson SH. The dark side of DNA repair. Elife. 2014; 3. 
146. Roberts SA, Sterling J, Thompson C, Harris S, Mav D, Shah R, Klimczak LJ, Kryukov GV, Malc E, Mieczkowski PA, Resnick MA, Gordenin DA. Clustered Mutations in Yeast and in Human Cancers Can Arise from Damaged Long Single-Strand DNA Regions. Molecular Cell. 2012; 46: 424-435.

147. Yang Y, Sterling J, Storici F, Resnick MA, Gordenin DA. Hypermutability of damaged single-strand DNA formed at double-strand breaks and uncapped telomeres in yeast Saccharomyces cerevisiae. PLoS Genetics. 2008; 4: e1000264.

148. Sakofsky CJ, Roberts SA, Malc E, Mieczkowski PA, Resnick MA, Gordenin DA, Malkova A. Break-induced replication is a source of mutation clusters underlying kataegis. Cell Reports. 2014; 7: 1640-1648.

149. Saini N, Ramakrishnan S, Elango R, Ayyar S, Zhang Y, Deem A, Ira G, Haber JE, Lobachev KS, Malkova A. Migrating bubble during break-induced replication drives conservative DNA synthesis. Nature. 2013; 502: 389392. 